\title{
DESARROLLANDO UNA PLATAFORMA MULTIMEDIAL PARA EL APRENDIZAJE DEL INGLÉS EN LAS CARRERAS DE INGENIERÍA DE LA UNIVERSIDAD CATÓLICA DE LA SANTÍSIMA CONCEPCIÓN
}

\section{DESIGNING A MULTIMEDIA PLATFORM FOR THE LEARNING OF ENGLISH IN THE ENGINEERING DEGREES AT UNIVERSIDAD CATÓLICA DE LA SANTÍSIMA CONCEPCIÓN}

\author{
Claudio Heraldo Díaz Larenas ${ }^{1}$ \\ Recibido el 5 de septiembre de 2007, aceptado el 13 de noviembre de 2007 \\ Received: September 5, 2007 Accepted: November 13, 2007
}

\begin{abstract}
RESUMEN
La adjudicación del proyecto MECESUP USC 0201 "Inglés como lengua extranjera en el pregrado: innovación de la enseñanza aprendizaje y mejoramiento de la competencia comunicativa en la Universidad Católica de la Santísima Concepción" permitió el desarrollo de una plataforma multimedial que tiene como objetivo apoyar el aprendizaje del idioma inglés de los estudiantes de las carreras de Ingeniería de la Universidad. Esta plataforma está compuesta de diferentes secciones que apuntan al desarrollo de la lectura, audición, escritura y expresión oral. Los estudiantes pueden, además, practicar vocabulario, gramática y pronunciación. El estado de avance de los estudiantes queda registrado en la plataforma, a fin de que los docentes puedan monitorear el trabajo de los estudiantes. Con la plataforma de aprendizaje se espera que los estudiantes desarrollen la habilidad del trabajo autónomo y, además, puedan mejorar su rendimiento en la asignatura de inglés.
\end{abstract}

Palabras clave: Inglés, plataforma multimedial, competencias.

\begin{abstract}
The government-funded project MECESUP USC 0201 "Inglés como lengua extranjera en el pregrado: innovación de la enseñanza aprendizaje y mejoramiento de la competencia comunicativa en la Universidad Católica de la Santísima Concepción" has led to the design of a multimedia platform whose main aim is to support engineering students'learning of English. The multimedia platform has got different sections that aim at developing reading, listening, writing and speaking skills. The students can also practice vocabulary, grammar, and pronunciation. Students' work progress remains registered in the platform so as to allow teachers to monitor students' work. Together with improving their language proficiency, it is expected that students can also develop autonomous learning strategies through their interaction with the platform.
\end{abstract}

Keywords: English, multimedia platform, competences.

\section{INTRODUCCIÓN}

El desarrollo y fortalecimiento de las habilidades de comunicación en inglés de los estudiantes de ingeniería de la Universidad Católica de la Santísima Concepción es uno de los fines del proyecto MECESUP USC 0201" "Inglés como lengua extranjera en el pregrado: innovación de la enseñanza aprendizaje y mejoramiento de la competencia comunicativa en la Universidad Católica de la Santísima Concepción" cuyo objetivo general es implementar innovaciones al proceso de enseñanza-aprendizaje tendientes a mejorar la competencia comunicativa del inglés de los estudiantes de Ingeniería de la Universidad, mediante el diseño de una plataforma multimedial 'online' que favorece el trabajo autónomo de los estudiantes en las habilidades de audición, expresión oral, lectura y escritura guiada.

Una de las razones que impulsaron la elaboración de este proyecto fue el hecho de que el campo laboral actual

\footnotetext{
1 Facultad de Educación. Universidad Católica de la Santísima Concepción. Alonso de Ribera 2850, Edificio Santo Tomás Moro, Campus San Andrés. Concepción, Chile. E-mail: cdiaz@ucsc.cl

2 Programa de Mejoramiento de la Calidad y Equidad de la Educación Superior (MECESUP).
} 
de cualquier carrera profesional requiere el manejo en inglés como una competencia profesional importante. Las innovaciones a la enseñanza aprendizaje de inglés de este proyecto se materializan mediante la incorporación de una metodología de enseñanza que favorece (1) el aprendizaje de habilidades de comprensión auditiva y expresión oral, (2) la creación de sistemas de apoyo a la enseñanza, (3) el diseño de recursos tecnológicos que favorezcan el aprendizaje remedial y autónomo del inglés, y (4) la implementación de un flujo curricular que permite incorporar la enseñanza-aprendizaje del idioma como un área de formación en cada carrera.

Este artículo informa acerca del desarrollo de un programa de inglés comunicativo de seis semestres que incorpora el uso de una plataforma multimedial 'online' como una herramienta de apoyo al desarrollo de habilidades lingüísticas por parte del estudiantado de Ingeniería. El artículo dará cuenta de la génesis del proyecto, su desarrollo y posterior evaluación.

\section{ANÁLISIS TEÓRICO}

\section{Antecedentes de la génesis del proyecto}

En el año 2003, la Universidad se encontraba en un proceso de determinación de su Proyecto Educativo Institucional ${ }^{3}$ y un Marco Curricular que tuviera como objetivo consensuar un nuevo diseño curricular que respondiera de mejor manera a los desafíos planteados a la educación superior y a las nuevas demandas de profesionales. Tal Proyecto Educativo considera una línea de formación general que contempla en los estudiantes el desarrollo de los lenguajes elementales sobre los cuales se cimentarán los posteriores aprendizajes. Concretamente, se consideraba el manejo del idioma materno, del inglés y de las aplicaciones comunes de informática e Internet.

La enorme oferta de carreras y universidades en la Octava Región obligaba a esta casa de estudios a buscar nuevas formas que otorgaran competencias diferenciadoras en sus egresados. En esta senda se embarcó la Universidad Católica de la Santísima Concepción al formular un proyecto de fortalecimiento e innovación de la enseñanza-aprendizaje del inglés en el pregrado con miras a entregar profesionales competitivos que pudieran insertarse rápidamente en el entorno laboral. Por lo tanto, este proyecto se sustentó en el tiempo por una opción institucional de atraer más estudiantes a sus aulas y entregar un producto competitivo.

\section{¿Cuál era el escenario de la Universidad antes de la implementación del nuevo programa de inglés comunicativo?}

Cada carrera tenía al año 2003 en promedio 3 semestres de inglés. Los cursos estaban orientados al desarrollo de la comprensión escrita de textos específicos de cada especialidad. La insuficiente exposición a la lengua extranjera hacía imposible la reorientación de dichos cursos, toda vez que la implementación de un enfoque comunicativo de la lengua requería del contacto sistemático de los sujetos con la lengua extranjera y de la incorporación de estrategias y recursos didácticos apropiados al nuevo enfoque.

Otro hecho de importancia era que en el transcurso de su vida universitaria, el estudiante se veía expuesto a diversas fuentes y estímulos externos cuyo idioma original era el inglés; sin embargo, no tenía una capacidad de respuesta y de uso eficiente de estos recursos. El hecho de no manejar esta lengua limitaba el acceso al conocimiento y, en consecuencia, su capacidad de reacción ante los avances científicos y tecnológicos del mundo de hoy. Lo anterior resultaba particularmente grave si se considera que el crecimiento económico y social de una nación está determinado, entre otros factores, por la capacidad de sus ciudadanos para acceder a nuevos conocimientos, para actualizarlos y recrearlos.

\section{Un diagnóstico inicial de la situación del inglés en la Universidad}

Estudios de encuestas de satisfacción y expectativas realizados al interior del proyecto MECESUP en el año 2003 a tres grupos diferentes: los estudiantes universitarios, los docentes universitarios y los empresarios como representantes del mundo laboral revelaron que estos actores consideraban que el idioma inglés era una herramienta imprescindible para toda persona que participara del proceso de globalización y deseara tener las capacidades y el conocimiento para acceder a la cultura anglosajona.

En la figura 1 se observa, por ejemplo, que el $56 \%$ de los estudiantes estaba muy de acuerdo con la idea de que el inglés era una herramienta necesaria para su inserción al medio laboral. A esto se sumaba un $21 \%$ de estudiantes que también se manifestaba en acuerdo con la importancia de dominar un idioma extranjero al momento de insertarse en el mercado laboral.

\footnotetext{
3 Decreto de Rectoría N 61/2003. Proyecto Educativo de la Universidad Católica de la Santísima Concepción.
} 


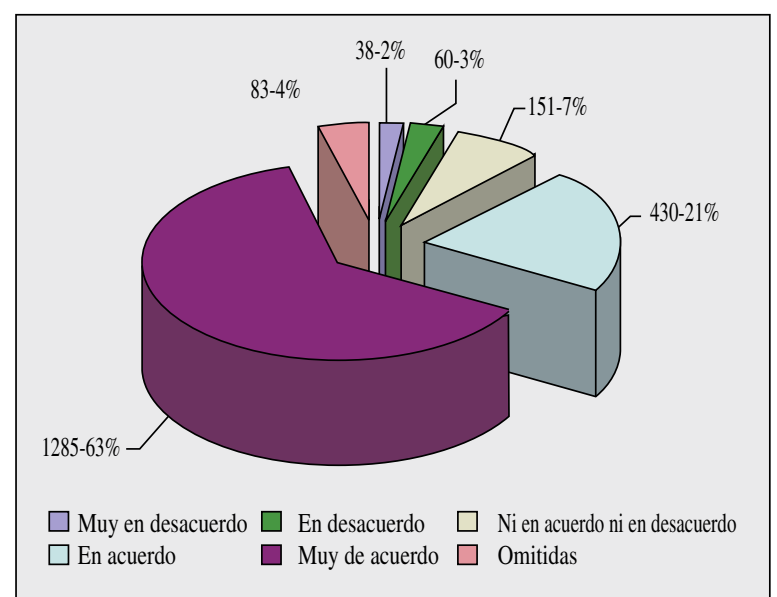

Figura 1. (N: 304) Importancia del idioma inglés para los estudiantes.

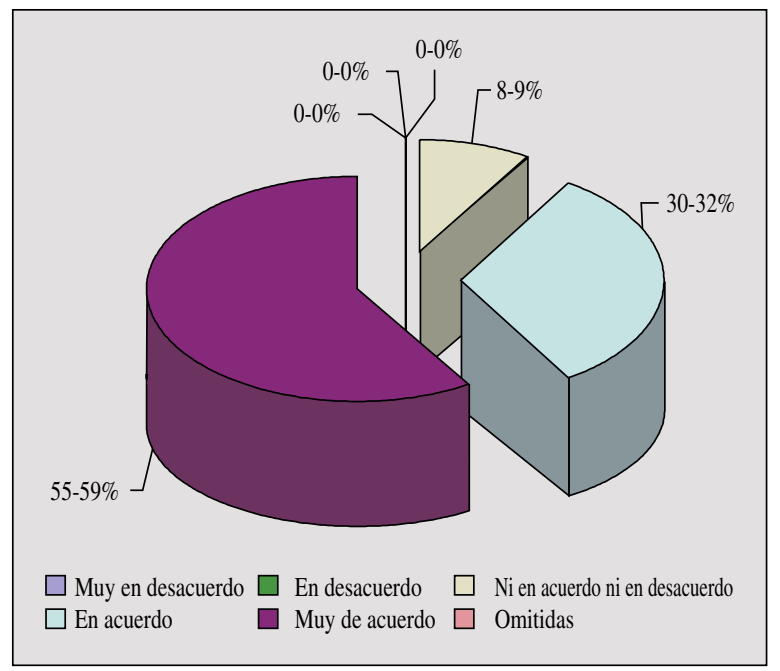

Figura 2. (N: 66) Importancia del idioma inglés para los docentes.

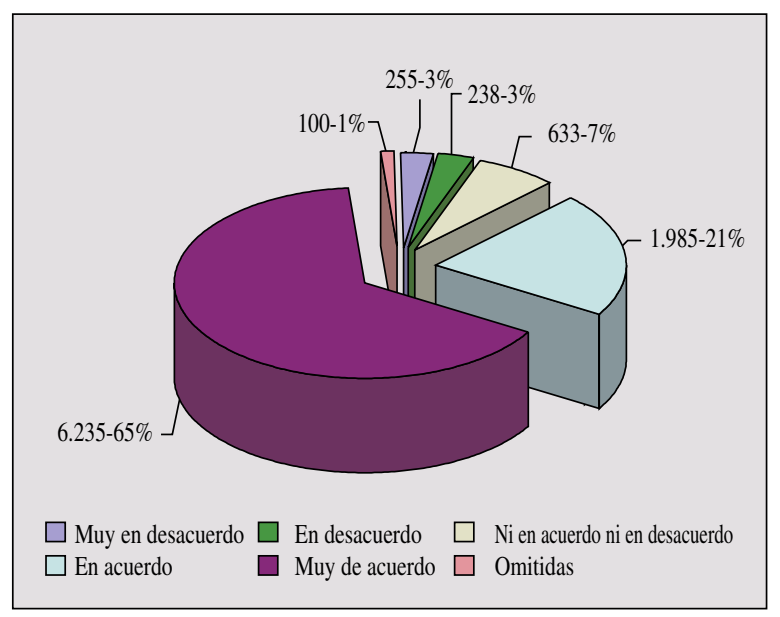

Figura 3. (N: 12) Importancia del idioma inglés para los empleadores.
De igual forma, los docentes coincidieron en alrededor de un $74 \%$ que el idioma inglés era una herramienta vital para que los estudiantes puedan acceder a la producción del nuevo conocimiento en sus respectivas disciplinas (ver figura 2).

Al observar la figura 3 ocurre lo mismo con la opinión de los empleadores, quienes en un $89 \%$ aprueban el manejo del idioma en el campo laboral.

Con toda esta información, el equipo del proyecto trabajó en el diseño de un nuevo programa de inglés comunicativo de seis semestres, cuyo objetivo principal era que los estudiantes de nuestra Universidad desarrollaran una competencia comunicativa oral a un nivel intermedio de complejidad.

Llama profundamente la atención que los grupos encuestados coincidan en que las habilidades lingüísticas más importantes al momento de aprender inglés son: (1) expresar opiniones personales y (2) mantener una conversación. Ambas habilidades insertas en la habilidad lingüísticas de expresión oral. En términos de frecuencia, también aparecieron otras habilidades lingüísticas como: participar en entrevistas, sintetizar información, describir un problema y ser capaz de presentarse en una situación formal.

Tabla 1. Necesidad de un nuevo programa de inglés.

\begin{tabular}{|l|l|}
\hline $\begin{array}{l}\text { Ingeniería } \\
\text { Comercial }\end{array}$ & $\begin{array}{l}\text { Que en el curso se practique más la } \\
\text { parte hablada tanto como la escrita. }\end{array}$ \\
\hline $\begin{array}{l}\text { Ingeniería } \\
\text { Comercial }\end{array}$ & $\begin{array}{l}\text { En general manejarse en el idioma } \\
\text { en distintas situaciones no sólo } \\
\text { profesionales. }\end{array}$ \\
\hline $\begin{array}{l}\text { Ingeniería } \\
\text { Comercial }\end{array}$ & ¡Bakan! Comunicación interactiva. \\
\hline $\begin{array}{l}\text { Ingeniería } \\
\text { Comercial }\end{array}$ & $\begin{array}{l}\text { Básicamente es necesario entender lo } \\
\text { que dicen en inglés y uno expresarse } \\
\text { satisfactoriamente. }\end{array}$ \\
\hline $\begin{array}{l}\text { Ingeniería } \\
\text { Comercial }\end{array}$ & $\begin{array}{l}\text { Que bueno que se preocupen de } \\
\text { nuestro desempeño profesional en el } \\
\text { futuro. }\end{array}$ \\
\hline $\begin{array}{l}\text { Ingeniería } \\
\text { Civil } \\
\text { Industrial }\end{array}$ & $\begin{array}{l}\text {..el idioma para mi carrera es una } \\
\text { herramienta fundamental en el } \\
\text { campo laboral que cada vez es más } \\
\text { competitivo. }\end{array}$ \\
\hline $\begin{array}{l}\text { Ingeniería } \\
\text { Civil } \\
\text { Industrial }\end{array}$ & $\begin{array}{l}\text { Todo lo que nos ayude a comprender } \\
\text { el idioma y lo que permita } \\
\text { relacionarnos (es bueno) }\end{array}$ \\
\hline $\begin{array}{l}\text { Ingeniería } \\
\text { Civil } \\
\text { Industrial }\end{array}$ & $\begin{array}{l}\text { Es importante que se aprenda a } \\
\text { conversar bien el idioma. }\end{array}$ \\
\hline $\begin{array}{l}\text { Ingeniería } \\
\text { Civil } \\
\text { Industrial }\end{array}$ & $\begin{array}{l}\text { Más horas de clases. El curso debe } \\
\text { poseer más clases prácticas. }\end{array}$ \\
\hline
\end{tabular}


En la misma línea del diagnóstico se recogieron algunas opiniones cualitativas (ver tabla 1) de los estudiantes de ingeniería Civil Industrial e Ingeniería Comercial que reflejaban el interés y la necesidad de contar con un nuevo programa de inglés que apuntara al desarrollo de la comprensión auditiva y la expresión como competencias fundamentales que deben tener los estudiantes egresados de esta Casa de Estudio.

También, se logró recoger opiniones de los académicos de las carreras de Ingeniería Comercial e Ingeniería Civil Industrial respecto a la importancia de incorporar el idioma inglés como una línea de cursos obligatorios al interior de los planes de estudio (ver tabla 2).

Tabla 2. Necesidad de un nuevo programa de inglés.

\begin{tabular}{|l|l|}
\hline $\begin{array}{l}\text { Ingeniería } \\
\text { Comercial }\end{array}$ & $\begin{array}{l}\text { Creo que el uso de una segunda } \\
\text { lengua hoy en el mundo de los } \\
\text { negocios exige muchos más... } \\
\text { debido a que las lecturas, } \\
\text { papers, diarios, etc, se basan } \\
\text { en la lingüística, gramática y } \\
\text { pronunciación buena del inglés. }\end{array}$ \\
\hline $\begin{array}{l}\text { Ingeniería Civil } \\
\text { Industrial }\end{array}$ & $\begin{array}{l}\text { Exponer temas profesionales en } \\
\text { forma oral, tipo congreso. }\end{array}$ \\
\hline
\end{tabular}

\section{¿Cuál son los objetivos del nuevo programa de inglés comunicativo?}

El nuevo programa de inglés apunta no sólo a interiorizar a los estudiantes en el sistema de la lengua que están aprendiendo sino que, por sobre todo, a capacitarlos para comunicarse en esa lengua. La competencia de comunicación [10], entendida como un saber que se actualiza en un hacer discursivo real, se caracteriza por una competencia organizacional o formal, que es el conocimiento de la organización de los elementos del código: fónico, gramatical, semántico, tanto en el nivel oracional como en el nivel textual (un saber), y una competencia discursiva, que le permite hacer funcionar el sistema en las comunicaciones reales (un saber hacer). Ambas se apoyan a su vez en una competencia mucho más amplia, que va más allá del lenguaje y de la comunicación, que es el ámbito sociocultural, y que se puede llamar competencia sociocultural, la que sustenta tanto la adquisición y la formación histórica del código, como el funcionamiento de los rituales de la comunicación" [1].

En este sentido, este programa tiene como objetivo desarrollar estrategias comunicativas superiores tales como: el uso de la inferencia, la búsqueda de información general y específica, la evaluación del discurso, la síntesis e interpretación de la información, la resolución de problemas, la enseñanza-aprendizaje de conceptos, el uso de estrategias metacognitivas y el desarrollo del pensamiento reflexivo y crítico. El desarrollo de todas estas estrategias permite optimizar el proceso de enseñanza-aprendizaje de la lengua, mediante la generación y utilización de recursos didácticos que apunten a desarrollar un mejor nivel de competencia comunicativa en inglés.

\section{El aporte de los recursos multimediales}

La incorporación de recursos e infraestructura multimedial ponen al servicio del estudiante y del proceso de enseñanzaaprendizaje diversas herramientas tecnológicas que ayudan a mejorar e innovar la enseñanza, con el fin último de producir aprendizajes significativos [8]. Además, permiten que el estudiante esté en contacto con textos auténticos (orales y escritos), esto es, en contacto con el discurso que es usado en forma cotidiana para comunicarse. La existencia de estos recursos tecnológicos permite, además, la implementación de programas de estrategias remediales de ayuda al aprendizaje de la lengua extranjera, tal como módulos tutoriales para estudiantes con dificultades.

La utilización de los recursos tecnológicos en la enseñanza de lenguas extranjeras permite, por una parte, que el sujeto realice tareas comunicativas que involucraran contacto directo con las lenguas tales como: sitios en la web, correos electrónicos, videos, audiciones y, por otra parte, que el sujeto amplíe su conocimiento de mundo mediante el acceso a nueva información [3].

En esta nueva perspectiva, el proceso didáctico se interpreta como un proceso comunicativo, por medio del cual estudiantes y profesores construyen conjuntamente el significado del saber disciplinario, interpretan y conceptualizan la experiencia. El profesor se convierte en un 'facilitador' del aprendizaje, quien enfatiza el proceso de enseñanza más que el resultado y promueve el aprendizaje cooperativo. Lo anterior se traduce en el uso de procedimientos que evalúen directamente el desempeño del estudiante en tareas "de la vida real".

Para lograr el desarrollo de la competencia comunicativa, el nuevo programa de inglés comunicativo sugiere una frecuencia de tres módulos semanales durante seis semestres, con un total de 384 horas cronológicas armonizadas con un fuerte programa de trabajo remedial independiente mediante el trabajo en una plataforma multimedial que responde a las expectativas de los alumnos de pregrado, de los docentes de las unidades académicas involucradas y de las necesidades de las empresas regionales y nacionales.

Ciertamente, la incorporación de tecnologías de vanguardia al proceso de enseñanza-aprendizaje del inglés no constituye una fortaleza por sí misma; sin embargo, es 
una herramienta fundamental para apoyar y desarrollar el aprendizaje del idioma [4]. Sin duda, estos recursos tecnológicos permiten mejorar e innovar las estrategias didácticas, puesto que el estudiante puede reforzar su aprendizaje de la lengua a su propio ritmo y desarrollar estrategias cognitivas y metacognitivas que lo vuelvan consciente de su aprendizaje.

De igual forma, la utilización de recursos tecnológicos en el proceso didáctico permite crear un ambiente favorable para la enseñanza-aprendizaje del idioma, de tal forma que se potencien en el estudiante todos aquellos componentes afectivos y de disposición que facilitaran el aprendizaje del inglés y garantizaran que el alumno tuviera un desempeño exitoso [2]. Lo anterior constituyó claramente una estrategia de retención, en la medida que permitió reducir los índices de reprobación y abandono de los estudios.

Al comenzar el siglo XXI, vemos cómo el uso del lenguaje está muy relacionado a la tecnología. Por lo tanto, el aprendizaje de una lengua utilizando este medio ha llegado a tener implicancias trascendentales para todos aquellos que estamos involucrados en los procesos que intervienen en el aprendizaje de una lengua extranjera [5]; de este modo, es necesario impregnarse de la naturaleza de las tareas de aprendizaje que los estudiantes pueden utilizar mediante la tecnología y cómo ellas pueden ser usadas para efectos de medición y evaluación.

La mayoría de los estudiantes de una lengua extranjera utilizan las nuevas tecnologías de la información, a lo menos, para elaborar documentos, mandar correos electrónicos y naturalmente para la búsqueda de información. Uno de los desafíos que surgen para los profesores que estamos involucrados en el proceso de aprendizaje de una lengua extranjera es el de utilizar y moldear las experiencias que tienen los estudiantes como usuarios tecnológicos y transformarlas en experiencias de aprendizaje.

\section{Desarrollo de la plataforma multimedial como apoyo para el programa de inglés}

En este sentido, la ejecución del proyecto MECESUP USC 0201 contempló el desarrollo de una plataforma multimedial de aprendizaje que permitiera desarrollar la competencia de aprendizaje autónomo de los estudiantes. Junto a las dos horas de trabajo presencial en el aula, se buscaba diseñar una plataforma multimedial que apoyara y fortaleciera el trabajo del estudiante y lo proveyera de las competencias remediales necesarias para subsanar cualquier dificultad en el aprendizaje.

Para esta tarea, un equipo de docentes de inglés e ingenieros y técnicos en informática emprendió la gran tarea de construir un ambiente de aprendizaje que contemplaba en su pantalla de presentación la identificación del usuario y su clave de acceso. También detallaba las instrucciones y los requerimientos técnicos necesarios para utilizar la plataforma desde cualquier lugar físico. Esta plataforma se llamó “The English Network” (Ver figura 4).

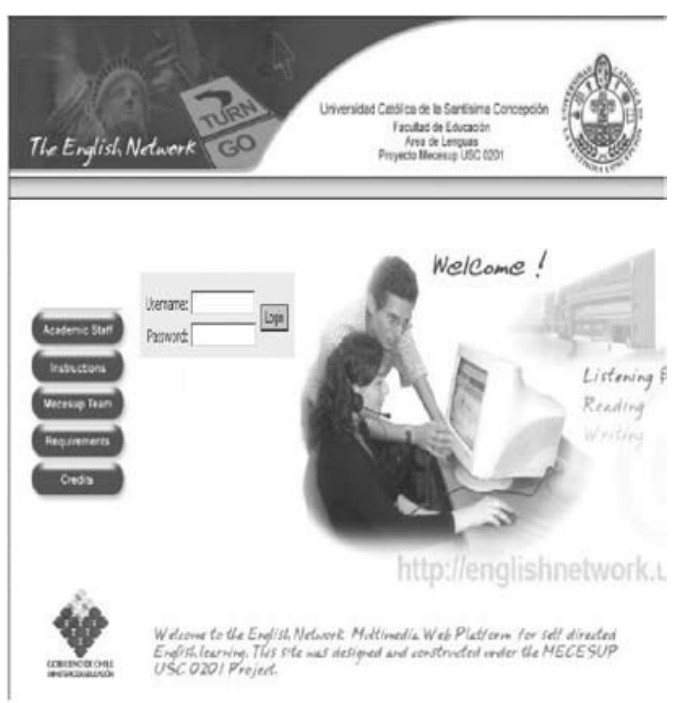

Figura 4. The English Network.

La pantalla que se muestra en la figura 7 describe el ambiente de aprendizaje que encontraba un estudiante al ingresar a uno de los niveles de los cursos de inglés. En total, la plataforma multimedial cuenta con cinco cursos que van desde el nivel básico hasta el nivel intermedio. Al interior del curso 1, por ejemplo, se indica la unidad temática, los objetivos de aprendizaje y las habilidades y sistemas lingüísticos en que puede trabajar el estudiante. Éste tiene la opción de trabajar en las habilidades de comprensión auditiva, comprensión de lectura, expresión escrita y expresión oral, esta última de manera guiada (ver figura 5).

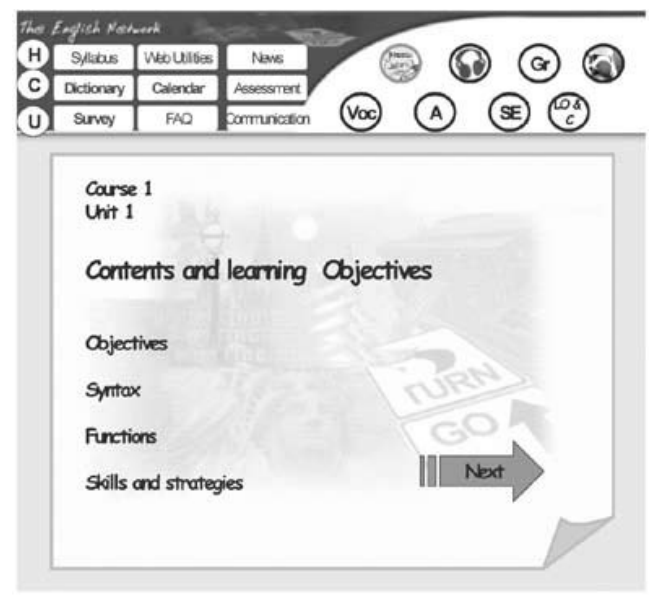

Figura 5. Ambiente de aprendizaje. 
La plataforma multimedial ofrece, además, otros recursos para el aprendizaje de los estudiantes. Por ejemplo, tienen la opción de usar algunos de los diccionarios 'online' o pueden contactarse con los técnicos, en caso que la plataforma presente algún problema técnico. Tiene, también una sección de evaluación que permite al docente monitorear el avance de los estudiantes y detectar algunos de los problemas de aprendizaje que el estudiante tenga.

Otro punto destacable es que la plataforma multimedial en el aprendizaje de una lengua tiene un rol diferente al que tenía en el pasado y por lo tanto los escenarios en el aprendizaje pueden ser ampliamente expandidos. El rango de modelos de interacción que está hoy en día disponible para los estudiantes es mucho mayor [6].

- Los estudiantes pueden autocorregirse o ser corregidos por el computador, profesor o pares.

- La comunicación se hace posible a través de dos o más usuarios en el mismo terminal, desde terminales geográficamente distantes o en un sentido interactivo entre el usuario y un video o audio.

- Los estudiantes pueden participar activamente en la lección, su interpretación y en las elecciones que ellos hacen.

- La flexibilidad en el aprendizaje está garantizada por la visión de los diseñadores del material de aprendizaje.

\section{Evaluación de la plataforma}

En el afán de evaluar la calidad de los aprendizajes que los estudiantes estaban logrando se ha aplicado semestralmente desde el año 2004 al 2006, una encuesta de satisfacción para evaluar el grado de aceptación que la plataforma multimedial tiene en los estudiantes. Esta encuesta se compone de tres grandes dimensiones: técnica, académica y de autoevaluación. A continuación se presentan los enunciados de la primera dimensión (tabla 3).

Esta dimensión obtiene un $83 \%$ de aceptación por parte de los estudiantes, quienes reconocen que el acceso y la navegación en la plataforma multimedial les resultan fáciles y amigables (Ver figura 6). También destacan la posibilidad que les otorga la plataforma para monitorear sus propios logros [9].
Tabla 3. Enunciados de la dimensión técnica.

\begin{tabular}{|l|}
\hline \multicolumn{1}{|c|}{ DIMENSIÓN TÉCNICA } \\
\hline (1) La forma de acceso a la plataforma es fácil \\
\hline $\begin{array}{l}\text { (2) La presentación de la plataforma es amigable (intuitivo } \\
\text { y fácil de usar) }\end{array}$ \\
\hline (3) La navegación interna de la plataforma es fácil \\
\hline (4) La parte gráfica es agradable a la vista \\
\hline (5) Es fácil navegar dentro de la plataforma \\
\hline (6) Me gusta tener acceso al informe de mi rendimiento \\
\hline (7) Los indicadores de los ejercicios son efectivos \\
\hline
\end{tabular}

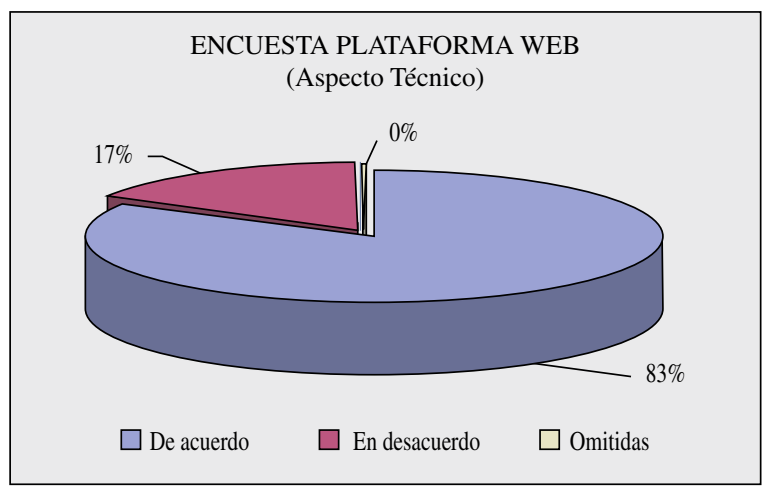

Figura 6. Porcentaje en la dimensión técnica.

Una segunda dimensión está caracterizada por las tareas de orden académico que los estudiantes pueden realizar al trabajar con la plataforma (ver tabla 4).

Consultados respecto de las opciones académicas que la plataforma multimedial les ofrece, en particular con el desarrollo de las actividades, los estudiantes manifiestan un grado de satisfacción alto, estimado en un $81 \%$ (ver figura 7).

Tabla 4. Enunciados de la dimensión académica.

\begin{tabular}{|l|}
\hline \multicolumn{1}{|c|}{ DIMENSIÓN ACADÉMICA } \\
\hline (1) Repetir una actividad hasta lograr su dominio \\
\hline (2) Ver videos auténticos y actuales \\
\hline (3) Ver actividades con imágenes \\
\hline (4) Escuchar textos auténticos en inglés \\
\hline (5) Trabajar sin profesor \\
\hline (6) Usar esta modalidad de aprendizaje como \\
motivación para aprender inglés \\
\hline (7) Aprender a mi propio ritmo \\
\hline (8) Realizar las actividades para consolidar lo \\
aprendido en clases \\
\hline (9) Poder usar el computador para aprender inglés \\
\hline
\end{tabular}




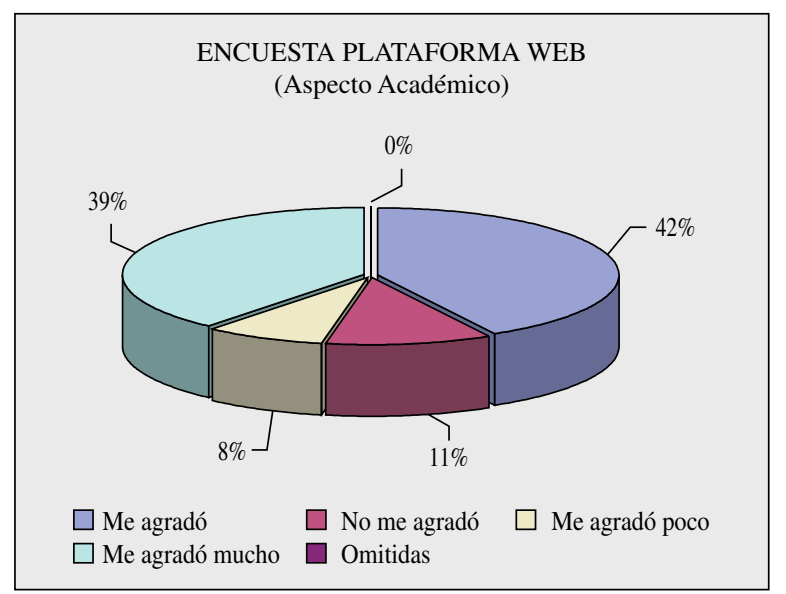

Figura 7. Porcentaje en la dimensión académica.

Finalmente, uno de los principales objetivos de la plataforma multimedial era justamente transformarse en una herramienta de autoevaluación para los estudiantes [7]. En esta línea, los estudiantes fueron consultados respecto al impacto que la plataforma ha tenido en su proceso de autoevaluación de su propio aprendizaje (ver tabla 5).

Con respecto a la autoevaluación, un $78 \%$ de los estudiantes valora a la plataforma como una herramienta de autoevaluación de sus propios aprendizajes (ver figura 8).

También como un indicador de evaluación del programa de inglés y de la efectividad de la plataforma multimedial, de un total de 94 estudiantes que egresaron de los seis semestres del programa de inglés, su rendimiento final por habilidad de la lengua fue el que se observa en la figura 9.

Tabla 5. Enunciados de la dimensión autoevaluación.

\begin{tabular}{|l|}
\hline \multicolumn{1}{|c|}{ DIMENSIÓN AUTOEVALUACIÓN } \\
\hline (1) Usar la gramática en forma correcta \\
\hline (2) Incrementar mi vocabulario \\
\hline (3) Desarrollar mi habilidad de comprensión auditiva \\
\hline (4) Mejorar mi habilidad de comprensión de lectura \\
\hline (5) Mejorar la escritura en inglés \\
\hline (6) Sentirme más segura/o al hablar en inglés \\
\hline (7) Aplicar en clase lo aprendido en la plataforma \\
\hline
\end{tabular}

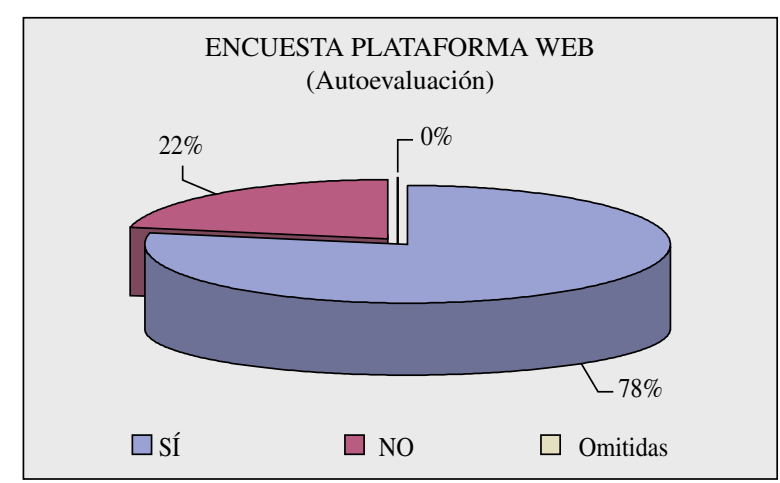

Figura 8. Porcentaje en la dimensión autoevaluación.

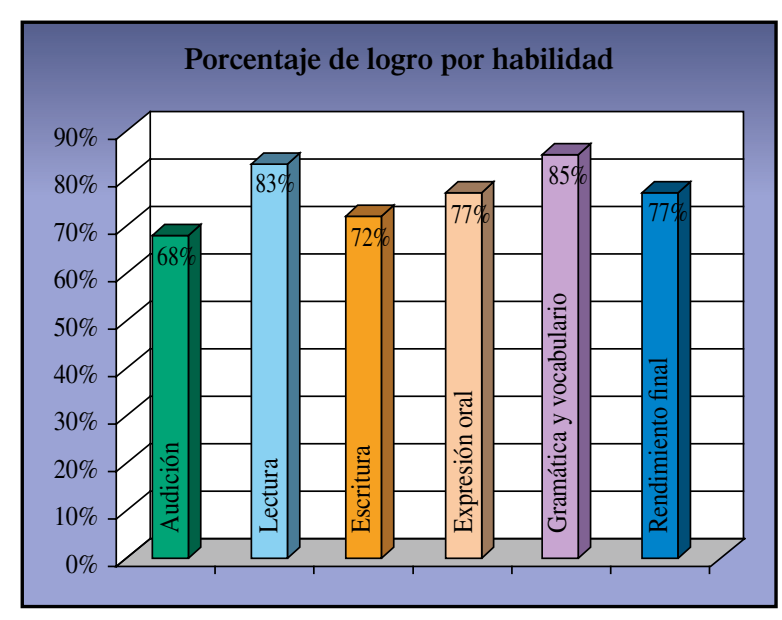

Figura 9. Porcentaje de logro por habilidad.

En la figura 9 se aprecia que las habilidades lingüísticas con mejores resultados fueron la lectura y la gramática y vocabulario, que alcanzaron un porcentaje de rendimiento por sobre el $80 \%$. Esto demuestra que la plataforma multimedial constituye una herramienta de apoyo significativa para las habilidades de comprensión escrita de la lengua. Las habilidades de audición y expresión oral se sitúan con un $68 \%$ y $77 \%$ respectivamente, que a pesar de no estar bajo el $60 \%$ exigido al estudiante, su aprendizaje requiere un apoyo mayor a través de la interacción que provee el docente en su contacto presencial con el estudiante al interior del aula. En suma, el rendimiento total del estudiante alcanza el $77 \%$.

\section{Otros diseños de plataformas}

El programa de Mejoramiento de la Calidad y Equidad de la Educación Superior (MECESUP) dependiente del Ministerio de Educación chileno asignó en sus concursos regulares recursos para la construcción de plataformas multimediales que potenciaran las habilidades autónomas de 
los estudiantes en el aprendizaje del inglés. Cada institución educativa construyó plataformas que respondieran a las necesidades comunicativas de sus estudiantes. Pionera en esta línea fue la Universidad Católica del Norte, seguida por otras experiencias similares en la Universidad de Concepción y Universidad Católica de Temuco. Todas ellas comparten en común la intención de desarrollar en sus educandos un segundo idioma, en este caso el inglés, con el fin de facilitar la inserción tanto de los estudiantes como docentes en la sociedad del conocimiento.

\section{CONCLUSIONES}

Para lograr una adecuada producción de material multimedial educativo es necesario considerar cuatro etapas o estadios no necesariamente lineales.

El primero de ellos tiene que ver con el diseño pedagógico, que conlleva a la planificación de las estrategias generales, según objetivos y metas e incluye:

- Definición de los objetivos de la plataforma

- Contenidos lingüísticos

- Prerrequisitos o cualidades de los destinatarios

- Ampliación y selección del método de enseñanza

- Paradigma de aprendizaje

- Estrategia instruccional

El diseño gráfico, segunda etapa, implica el diseño de pantalla que corresponde a la aparición visual del material de aprendizaje y su estructura espacio-temporal. La implementación o desarrollo de la plataforma involucra las etapas de codificación lógica del material pedagógico y la programación del software, transformándose esto en la tercera etapa. Una cuarta etapa se relaciona con la evaluación. En este sentido, cabe recordar que los estudiantes usan los computadores en diferentes formas, por lo tanto los profesores, investigadores y los productores de plataformas multimediales tienen que estar conscientes de cuáles actividades de aprendizaje de lenguas asistidas por computador son las más adecuadas.

Los computadores han sido considerados como ideales para la instrucción individual, especialmente hoy en día cuando ellos están al alcance económico de las instituciones educacionales. Diversos programas computacionales educativos siguen siendo desarrollados en los Estados Unidos y el Reino Unido para estos fines. Por esta razón, cobra vigencia el hecho de que las instituciones de educación terciaria inicien el desarrollo de plataformas multimediales que respondan a las necesidades comunicativas de sus propios educandos $\mathrm{y}$, a su vez, respondan al contexto sociocultural de los usuarios.

En suma, cualquier programa computacional cuyas características estructurales y funcionales permitan servir de apoyo a la enseñanza, el aprendizaje y la administración educacional debe integrar secuencial y fluidamente los procesos de diseño (la planificación pedagógica del programa); desarrollo (materialización del diseño mediante un programa determinado) y evaluación (validación interna y externa del programa en cuanto a su consistencia, efectividad y pertinencia educativa como grado de utilización de las ventajas del computador).

\section{AGRADECIMIENTOS}

Esta publicación ha sido posible gracias al financiamiento de la Dirección de Investigación de la Universidad Católica de la Santísima Concepción para asistir al Congreso Internacional de Profesores de Inglés, en la ciudad de Seattle, Estados Unidos, durante el mes de marzo de 2007.

\section{REFERENCIAS}

[1] G. Álvarez y N. Díaz, N. "Conceptos lingüísticos para el profesor de idiomas". Proyecto de Desarrollo de Docencia. Universidad de Concepción. Vol. 1 No 1, pp. 100-104. Concepción, Chile. 1996.

[2] R. Bothelio and B. Tomlinson. "Discover English". Oxford: Macmillan Heinemann. Oxford, Great Britain. Vol. 3 No 2, pp. 55-78. 1995.

[3] D. Brown. "Teaching by principles. An interactive approach to language pedagogy". Longman. New York, USA. Vol. $1 \mathrm{~N}^{\mathrm{o}}$ 3, pp. 23-34. 2001.

[4] S. Burgess and K. Hed. "How to teach for exams". Longman. London, Great Britain. Vol. $2 \mathrm{~N}^{\mathrm{o}} 1$, pp. 57-66, 2005.

[5] R. Carter and D. Nunan. "Teaching English to speakers of other languages". Cambridge University Press. Cambridge, Great Britain. Vol. $3 \mathrm{~N}^{\mathrm{o}} 1$, pp. 43-54. 2001

[6] M. Celce-Murcia. "Teaching English as a second or foreign language”. Heinle and Heinle. Boston, USA. Vol. $4 \mathrm{~N}^{\mathrm{o}}$ 1, pp. 88-98. 2001. 
[7] A. Chamot, S. Barhardt., E. Beard, P. Dinary and J. Robbins. "The learning strategies handbook". Longman. New York, USA. Vol. 2 No 1, pp. 55-65. 1999.

[8] D. Fernández. "Interactive computer-mediated teacher training: Supporting change in a process of educational reorientation”. Santa Fe, Argentina. Vol. $1 \mathrm{~N}^{\mathrm{o}}$ 1, pp. 29-33. 2000.
[9] J. Harmer. "The practice of English language". Longman. London, Great Britain. Vol. $3 \mathrm{~N}^{\mathrm{o}} 1$, pp. 12-34. 2001.

[10] D. Hymes. "On communicative competence". Penguin. London, Great Britain. Vol. $1 \mathrm{~N}^{\circ} 1$, pp. 43-77. 1972. 